\title{
First Measurement of Inclusive Muon Neutrino Charged Current Differential Cross Sections on Argon at $E_{\nu} \sim 0.8 \mathrm{GeV}$ with the MicroBooNE Detector
}

P. Abratenko, ${ }^{17}$ C. Adams, ${ }^{10}$ M. Alrashed, ${ }^{12}$ R. An, ${ }^{11}$ J. Anthony, ${ }^{3}$ J. Asaadi,${ }^{29}$ A. Ashkenazi, ${ }^{16}$ M. Auger, ${ }^{1}$ S. Balasubramanian, ${ }^{34}$ B. Baller, ${ }^{9}$ C. Barnes,${ }^{17}$ G. Barr, ${ }^{20} \mathrm{M}$. Bass, ${ }^{2}$ F. Bay, ${ }^{30}$ A. Bhat,${ }^{26} \mathrm{~K}$. Bhattacharya,${ }^{21}$ M. Bishai, ${ }^{2}$ A. Blake, ${ }^{13}$ T. Bolton, ${ }^{12}$ L. Camilleri, ${ }^{7}$ D. Caratelli, ${ }^{9}$ I. Caro Terrazas, ${ }^{6}$ R. Carr, ${ }^{16}$ R. Castillo Fernandez, ${ }^{9}$ F. Cavanna, ${ }^{9}$ G. Cerati, ${ }^{9}$ Y. Chen, ${ }^{1}$ E. Church, ${ }^{21}$ D. Cianci, ${ }^{7}$ E. O. Cohen, ${ }^{27}$ G. H. Collin, ${ }^{16}$ J. M. Conrad, ${ }^{16}$ M. Convery, ${ }^{24}$ L. Cooper-Troendle, ${ }^{34}$ J. I. Crespo-Anadón, ${ }^{7}$ M. Del Tutto, ${ }^{20}$ A. Devitt, ${ }^{13}$ A. Diaz, ${ }^{16}$ L. Domine, ${ }^{24}$ K. Duffy, ${ }^{9}$ S. Dytman, ${ }^{22}$ B. Eberly, ${ }^{8}$ A. Ereditato, ${ }^{1}$ L. Escudero Sanchez, ${ }^{3}$ J. Esquivel, ${ }^{26}$ J. J. Evans, ${ }^{15}$ R. S. Fitzpatrick, ${ }^{17}$ B. T. Fleming,${ }^{34}$ D. Franco ${ }^{34}$ A. P. Furmanski, ${ }^{15}$ D. Garcia-Gamez, ${ }^{15}$ V. Genty, ${ }^{7}$ D. Goeldi, ${ }^{1}$ S. Gollapinni, ${ }^{28}$ O. Goodwin,,${ }^{15}$ E. Gramellini, ${ }^{34,9}$ H. Greenlee, ${ }^{9}$ R. Grosso, ${ }^{5}$ L. Gu ${ }^{32}$ W. Gu, ${ }^{2}$ R. Guenette, ${ }^{10}$ P. Guzowski, ${ }^{15}$ A. Hackenburg, ${ }^{34}$ P. Hamilton, ${ }^{26}$ O. Hen, ${ }^{16}$ C. Hill, ${ }^{15}$ G. A. Horton-Smith, ${ }^{12}$ A. Hourlier, ${ }^{16}$ E.-C. Huang, ${ }^{14}$ C. James,,${ }^{9}$ J. Jan de Vries,${ }^{3}$ X. Ji, ${ }^{2}$ L. Jiang, ${ }^{22}$ R. A. Johnson, ${ }^{5}$ J. Joshi, ${ }^{2}$ H. Jostlein, ${ }^{9}$ Y.-J. Jwa, ${ }^{7}$ G. Karagiorgi, ${ }^{7}$ W. Ketchum, ${ }^{9}$ B. Kirby, ${ }^{2}$ M. Kirby, ${ }^{9}$ T. Kobilarcik, ${ }^{9}$ I. Kreslo, ${ }^{1}$ I. Lepetic, ${ }^{11}$ Y. Li,${ }^{2}$ A. Lister, ${ }^{13}$ B. R. Littlejohn, ${ }^{11}$ S. Lockwitz, ${ }^{9}$ D. Lorca, ${ }^{1}$ W. C. Louis, ${ }^{14}$ M. Luethi, ${ }^{1}$ B. Lundberg, ${ }^{9}$ X. Luo, ${ }^{34}$ A. Marchionni, ${ }^{9}$ S. Marcocci, ${ }^{9}$ C. Mariani, ${ }^{32}$ J. Marshall,, 33 J. Martin-Albo, ${ }^{10}$ D. A. Martinez Caicedo, ${ }^{1,25}$ K. Mason, ${ }^{31}$ A. Mastbaum, ${ }^{4}$ V. Meddage, ${ }^{12}$ T. Mettler, ${ }^{1}$ J. Mills, ${ }^{31}$ K. Mistry, ${ }^{15}$ A. Mogan, ${ }^{28}$ J. Moon, ${ }^{16}$ M. Mooney, ${ }^{6}$ C. D. Moore,${ }^{9}$ J. Mousseau, ${ }^{17}$ M. Murphy, ${ }^{32}$ R. Murrells,${ }^{15}$ D. Naples, ${ }^{22}$ P. Nienaber, ${ }^{23}$ J. Nowak, ${ }^{13}$ O. Palamara, ${ }^{9}$ V. Pandey, ${ }^{32}$ V. Paolone, ${ }^{22}$ A. Papadopoulou, ${ }^{16}$ V. Papavassiliou, ${ }^{18}$ S. F. Pate, ${ }^{18}$ Z. Pavlovic, ${ }^{9}$ E. Piasetzky, ${ }^{27}$ D. Porzio, ${ }^{15}$ G. Pulliam, ${ }^{26}$ X. Qian, ${ }^{2}$ J. L. Raaf, ${ }^{9}$ A. Rafique, ${ }^{12}$ L. Ren, ${ }^{18}$ L. Rochester, ${ }^{24}$ H. E. Rogers, ${ }^{6}$ M. Ross-Lonergan, ${ }^{7}$ C. Rudolf von Rohr, ${ }^{1}$ B. Russell, ${ }^{34}$ G. Scanavini, ${ }^{34}$ D. W. Schmitz, ${ }^{4}$ A. Schukraft, ${ }^{9}$ W. Seligman, ${ }^{7}$ M. H. Shaevitz,

R. Sharankova, ${ }^{31}$ J. Sinclair, ${ }^{1}$ A. Smith, ${ }^{3}$ E. L. Snider, ${ }^{9}$ M. Soderberg, ${ }^{26}$ S. Söldner-Rembold, ${ }^{15}$ S. R. Soleti, ${ }^{20,10}$

P. Spentzouris, ${ }^{9}$ J. Spitz, ${ }^{17}$ M. Stancari, ${ }^{9}$ J. St. John,,${ }^{9}$ T. Strauss, ${ }^{9}$ K. Sutton, ${ }^{7}$ S. Sword-Fehlberg, ${ }^{18}$ A. M. Szelc, ${ }^{15}$ N. Tagg, ${ }^{19}$ W. Tang, ${ }^{28}$ K. Terao, ${ }^{24}$ M. Thomson, ${ }^{3}$ R. T. Thornton, ${ }^{14}$ M. Toups, ${ }^{9}$ Y.-T. Tsai, ${ }^{24}$ S. Tufanli, ${ }^{34}$ T. Usher, ${ }^{24}$ W. Van De Pontseele, ${ }^{20,10}$ R. G. Van de Water, ${ }^{14}$ B. Viren, ${ }^{2}$ M. Weber, ${ }^{1}$ H. Wei, ${ }^{2}$ D. A. Wickremasinghe,${ }^{22}$ K. Wierman, ${ }^{21}$ Z. Williams, ${ }^{29}$ S. Wolbers, ${ }^{9}$ T. Wongjirad, ${ }^{31}$ K. Woodruff, ${ }^{18}$ W. Wu, ${ }^{9}$ T. Yang,${ }^{9}$ G. Yarbrough, ${ }^{28}$ L. E. Yates, ${ }^{16}$ G. P. Zeller, ${ }^{9}$ J. Zennamo, ${ }^{9}$ and C. Zhang ${ }^{2}$

\section{(MicroBooNE Collaboration)}

\author{
${ }^{1}$ Universität Bern, Bern CH-3012, Switzerland \\ ${ }^{2}$ Brookhaven National Laboratory (BNL), Upton, New York 11973, USA \\ ${ }^{3}$ University of Cambridge, Cambridge CB3 OHE, United Kingdom \\ ${ }^{4}$ University of Chicago, Chicago, Illinois 60637, USA \\ ${ }^{5}$ University of Cincinnati, Cincinnati, Ohio 45221, USA \\ ${ }^{6}$ Colorado State University, Fort Collins, Colorado 80523, USA \\ ${ }^{7}$ Columbia University, New York, New York 10027, USA \\ ${ }^{8}$ Davidson College, Davidson, North Carolina 28035, USA \\ ${ }^{9}$ Fermi National Accelerator Laboratory (FNAL), Batavia, Illinois 60510, USA \\ ${ }^{10}$ Harvard University, Cambridge, Massachusetts 02138, USA \\ ${ }^{11}$ Illinois Institute of Technology (IIT), Chicago, Illinois 60616, USA \\ ${ }^{12}$ Kansas State University (KSU), Manhattan, Kansas 66506, USA \\ ${ }^{13}$ Lancaster University, Lancaster LA1 4YW, United Kingdom \\ ${ }^{14}$ Los Alamos National Laboratory (LANL), Los Alamos, New Mexico 87545, USA \\ ${ }^{15}$ The University of Manchester, Manchester M13 9PL, United Kingdom \\ ${ }^{16}$ Massachusetts Institute of Technology (MIT), Cambridge, Massachusetts 02139, USA \\ ${ }^{17}$ University of Michigan, Ann Arbor, Michigan 48109, USA \\ ${ }^{18}$ New Mexico State University (NMSU), Las Cruces, New Mexico 88003, USA \\ ${ }^{19}$ Otterbein University, Westerville, Ohio 43081, USA \\ ${ }^{20}$ University of Oxford, Oxford OXI 3RH, United Kingdom \\ ${ }^{21}$ Pacific Northwest National Laboratory (PNNL), Richland, Washington 99352, USA \\ ${ }^{22}$ University of Pittsburgh, Pittsburgh, Pennsylvania 15260, USA \\ ${ }^{23}$ Saint Mary's University of Minnesota, Winona, Minnesota 55987, USA \\ ${ }^{24}$ SLAC National Accelerator Laboratory, Menlo Park, California 94025, USA
}




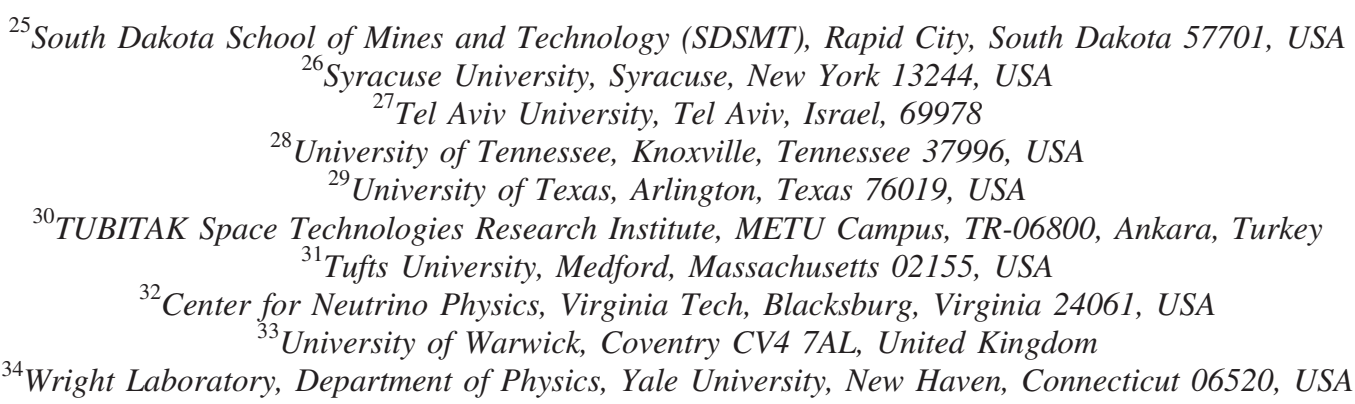

(Received 8 June 2019; revised manuscript received 6 August 2019; published 23 September 2019)

We report the first measurement of the double-differential and total muon neutrino charged current inclusive cross sections on argon at a mean neutrino energy of $0.8 \mathrm{GeV}$. Data were collected using the MicroBooNE liquid argon time projection chamber located in the Fermilab Booster neutrino beam and correspond to $1.6 \times 10^{20}$ protons on target of exposure. The measured differential cross sections are presented as a function of muon momentum, using multiple Coulomb scattering as a momentum measurement technique, and the muon angle with respect to the beam direction. We compare the measured cross sections to multiple neutrino event generators and find better agreement with those containing more complete treatment of quasielastic scattering processes at low $Q^{2}$. The total flux integrated cross section is measured to be $0.693 \pm 0.010$ (stat) \pm 0.165 (syst) $\times 10^{-38} \mathrm{~cm}^{2}$.

DOI: 10.1103/PhysRevLett.123.131801

Current and next generation precision neutrino oscillation experiments aim to probe beyond standard model physics, such as $C P$ violation in the lepton sector and sterile neutrinos. These experiments measure the oscillation probability which, in the experimental setup, is convolved with models of the neutrino interaction cross section and kinematics of secondary leptons and hadrons emerging from the neutrino's interaction. This link is complicated by the existence of nuclear effects and final-state interactions, which to date cannot be modeled precisely, in particular for heavy target nuclei typically used in modern neutrino experiments. Many future experiments, including DUNE [1-3] and the SBN [4] program, employ liquid argon time projection chambers (LArTPCs) as detectors. As a consequence, neutrino-argon cross section measurements have paramount importance, especially given the relative scarcity of neutrino-argon data $[5,6]$.

We present the first $\nu_{\mu}$ charged current (CC) inclusive double-differential (in muon momentum and scattering angle) cross section measurement on argon. Neutrinos in the same $\sim 1 \mathrm{GeV}$ energy range will be studied by the SBND and ICARUS experiments, and this is the energy where DUNE is very sensitive to precise measurements of oscillation parameters as $C P$ violating effects are maximized at this energy and DUNE's baseline. The inclusive

Published by the American Physical Society under the terms of the Creative Commons Attribution 4.0 International license. Further distribution of this work must maintain attribution to the author(s) and the published article's title, journal citation, and DOI. Funded by SCOAP.
$\mathrm{CC}$ process, in which only the outgoing muon is required to be reconstructed, comprises multiple interaction processes and is dominantly quasielastic scattering in the case of MicroBooNE [7]. Inclusive measurements are particularly important as the clear signal definition allows a straightforward comparison to theory models and other experiments. They are also the foundation for studies of more complex event topologies involving detection of hadrons in the final state. With the fully active and high-resolution MicroBooNE LArTPC detector, the outgoing muon phase space can be probed with full acceptance in both angle and momentum for the first time. The momentum of the outgoing muon is measured by using multiple Coulomb scattering (MCS) [8], thus allowing the analysis sample to include both exiting and contained muons.

The MicroBooNE detector has 85 tons of liquid argon active mass and is located along the Booster Neutrino Beam (BNB) at Fermilab, $463 \mathrm{~m}$ from the target. The BNB consists primarily of $\nu_{\mu}(93.6 \%)$ with energy from a few tens of $\mathrm{MeV}$ to $\sim 2 \mathrm{GeV}$. If neutrinos interact in the MicroBooNE detector [9], charged particles traverse a volume of highly pure liquid argon leaving trails of ionization electrons along their paths, and also create prompt ultraviolet scintillation photons. Ionization electrons drift horizontally and transverse to the neutrino beam direction in an electric field of $273 \mathrm{~V} / \mathrm{cm}$ to a system of three anode wire planes located $2.5 \mathrm{~m}$ from the cathode plane and are detected by electronics immersed in the liquid argon [10]. Scintillation photons are observed by 32 photomultipliers (PMTs) [11].

The data used in this analysis are taken from an exposure of $1.6 \times 10^{20}$ protons on target (POT), after applying data 
quality criteria for the beam and detector operating conditions. This corresponds to a four-month exposure, from February to July 2016. Two different data streams are used in this analysis: an on-beam data sample, triggered by BNB neutrino spills, and an off-beam data sample, taken during periods when no beam was received. The off-beam data sample is used for a measurement of cosmic ray (CR) backgrounds, which is important because the MicroBooNE detector operates on Earth's surface.

The flux of neutrinos at the MicroBooNE detector is simulated using the framework built by the MiniBooNE Collaboration [12]. Neutrino interactions in the MicroBooNE detector are simulated using the GENIE event generator [13], which generates the primary neutrino interaction inside the nucleus, the production of all finalstate particles in the nucleus (hadronization), and the transport and rescattering of the final-state particles through the nucleus (final-state interactions). CRs crossing the detector volume within the readout window of neutrino events are simulated with CORSIKA [14]. Particle propagation is based on GEANT4 [15], while the simulation of the MicroBooNE detector is performed in the LARSOFT framework [16] and includes the generation of wire signals and the modeling of scintillation light in the PMTs.

Data processing begins with a requirement that PMT activity occurs in coincidence with the arrival of neutrinos. This PMT trigger results in a negligible loss of signal. TPC waveforms originate from drift electrons inducing bipolar signals on the first two wire planes and a unipolar signal on the last plane, which collects the electrons. A noise filtering algorithm removes inherent and electronic noise [10], and the signals are deconvolved to a Gaussian to further eliminate detector artifacts [17]. Individual signal waveforms are identified as hits and are sorted spatially to form clusters. Clusters are matched across planes and identified as tracklike or showerlike by the Pandora multialgorithm pattern recognition framework [18]. Optical reconstruction combines correlated PMT waveforms across the detector into flashes.

A series of algorithms is used to identify and remove CRs. These algorithms identify tracks that traverse the detector from top to bottom, adding optical information to identify CRs that enter from the anode or cathode planes. Stopping muon tracks originating outside the detector are identified as CRs either by their Bragg peak or by their Michel decay.

This analysis makes use of the optical system to reduce the high CR rate by more than 3 orders of magnitude. The observed reconstructed optical neutrino candidate flash, which is the spatial intensity distribution of scintillation light arriving at the PMTs behind the anode plane during the $1.6 \mu$ s beam window, is compared to a light prediction made from TPC tracks and showers originating from a common vertex found in the same $\sim 1 \mathrm{~ms}$ duration event. The best matched pair (if any) identifies the neutrino interaction and its secondary tracks and showers within the event. Calorimetric information [19] in the form of a truncated mean value of the deposited charge per unit length, $d Q / d x$, and track length are used to discriminate muons from protons. The candidate interaction must contain a track that has a measured $d Q / d x$ compatible with a muon. In cases where there are multiple particles originating from the same vertex (predicted to occur in $70 \%$ of the neutrino events), only one such muonlike track is identified by selecting the longest track. The purity of selecting a true muon is $95 \%$. Several algorithms ensure the quality of the fitted track by limiting the allowed spatial dispersion of the reconstructed hits with respect to the track hypothesis.

The momentum of the muon is measured using multiple Coulomb scattering. Here, the magnitude of the momentum is a fit parameter that describes the scattering pattern of the track [8]. The strength of this algorithm is that it can estimate the muon momentum for muon tracks spatially contained in the detector as well as exiting tracks, which is important given that only $30 \%$ of neutrino-induced muons are spatially contained. In addition, the measured momentum using range is used to identify and exclude misreconstructed tracks by comparing it to the MCS momentum. The two momentum estimates would disagree if the reconstructed track is incomplete or inaccurate.

Figure 1 shows the measured versus generated muon momentum $p_{\mu}$ and the measured versus generated $\cos \theta_{\mu}$ for simulated events, where $\theta_{\mu}$ is the muon angle with respect to the incident neutrino beam direction. There is a possibility for tracks to be misreconstructed with the opposite direction [18]. The impact is strongest in the two backwards bins, $\cos \theta_{\mu} \in[-1,-0.5)$ and $\cos \theta_{\mu} \in[-0.5,0)$, where only $46 \%$ and $56 \%$ of events come from the same bin they were generated in, respectively. The other events are actually forward going but get reconstructed with the opposite direction [18]. This effect is included in the smearing matrix; therefore, the muon kinematic distributions of data and simulation remain comparable with each other.

The final selected sample contains 27200 events. The signal selection efficiency, measured in simulation, is 57.2\%. The selection accepts events across the entire angular phase space. The purity of the final selection is $50.4 \%$. The efficiency and purity are relatively flat as a function of muon kinematics and the number of final-state particles. Different interaction processes have approximately the same efficiency. The main backgrounds in this analysis are (i) CRs that overlap in time with the beam spill and trigger the readout (estimated to be $29.1 \%$ of all selected events), (ii) CRs overlaid with neutrino interactions in which the cosmic muon was misidentified as coming from a neutrino interaction (6.4\%), (iii) neutrinos that interact outside the fiducial volume with an entering track selected as the muon candidate (7.6\%), (iv) events in which a neutrino interacts outside the cryostat but the 


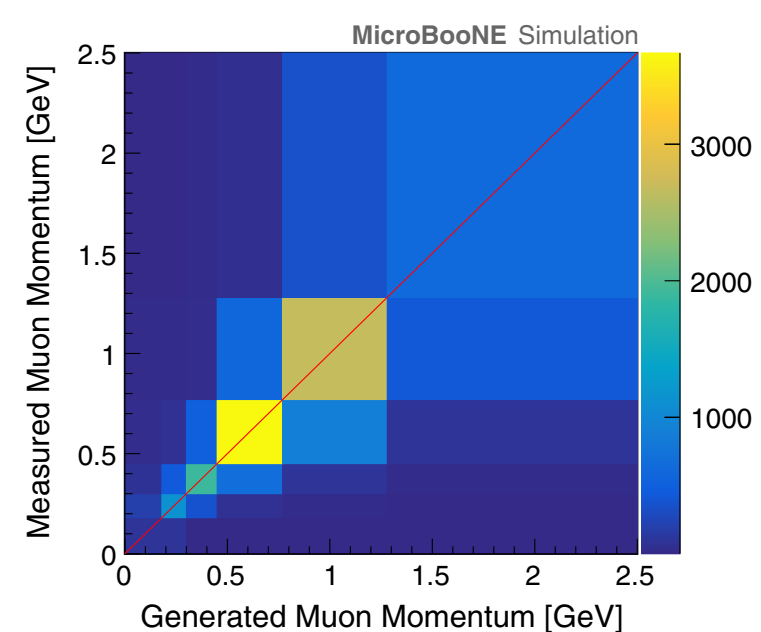

(a)

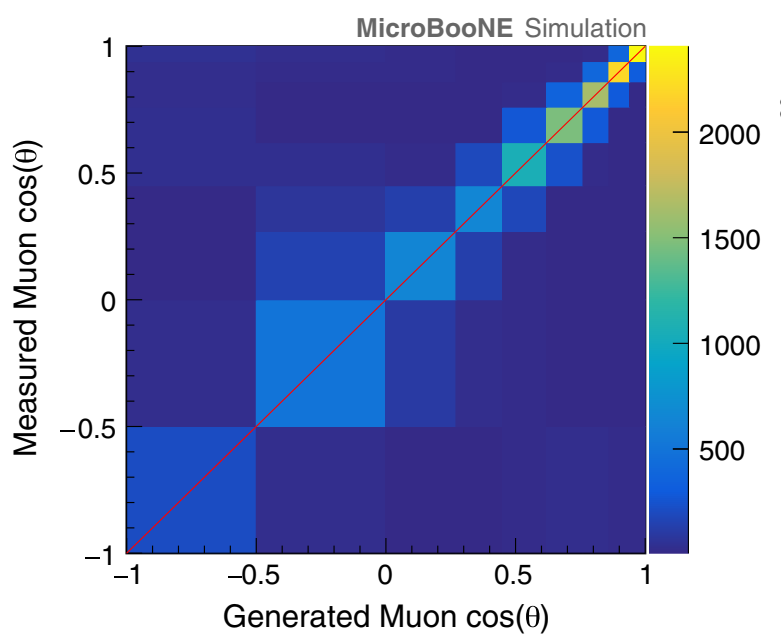

(b)

FIG. 1. Comparison of the generated versus measured muon momentum (a) and cosine of the muon angle (b) for the simulated and selected $\nu_{\mu}$ CC events, showing a 10\%-15\% momentum resolution and few-degree angular resolution. The binning is the same as used in the cross section extraction.

muon enters the TPC and is selected (here called "dirt" interactions) (4.4\%), (v) neutral current interactions where a final-state particle is misidentified as a muon (1.6\%), (vi) beam intrinsic muon antineutrino interactions $(0.4 \%)$, and (vii) beam intrinsic electron (anti)neutrino interactions $(0.1 \%)$. The largest background (i) is measured with offbeam data and subtracted from the on-beam data. The offbeam data sample has twice the statistics of the on-beam data. Other backgrounds are estimated from simulation. The accuracy of the detector modeling has been verified by looking at selected event distributions of variables not affected by the neutrino interaction physics, e.g., the interaction points in the detector, where we have good data to simulation agreement.

This analysis measures the double-differential $\nu_{\mu} \mathrm{CC}$ cross section on argon as a function of the muon momentum $p_{\mu}$ (measured using MCS) and the cosine of the muon angle $\theta_{\mu}$ with respect to the beam direction. The fluxintegrated, double-differential cross section measured in bin $i$ is defined as

$$
\left(\frac{d^{2} \sigma}{d p_{\mu} d \cos \theta_{\mu}}\right)_{i}=\frac{N_{i}-B_{i}}{\tilde{\epsilon}_{i} T \Phi_{\nu_{\mu}}\left(\Delta p_{\mu} \Delta \cos \theta_{\mu}\right)_{i}},
$$

where $N_{i}, B_{i}$, and $\tilde{\epsilon_{i}}$ are the number of selected data events, the expected background events, and the detection efficiency in bin $i .\left(\Delta p_{\mu} \Delta \cos \theta_{\mu}\right)_{i}$ is the $i$ th bin area. $T$ and $\Phi_{\nu_{\mu}}$ are the number of target nucleons, and the integrated BNB muon neutrino flux from 0 to $10 \mathrm{GeV}$. The total integrated BNB $\nu_{\mu}$ flux in neutrino mode running, corresponding to $1.6 \times 10^{20}$ POT, is $\Phi_{\nu_{\mu}}=1.16 \times 10^{11} \nu_{\mu} / \mathrm{cm}^{2}$, and its mean neutrino energy is $823 \mathrm{MeV}$. The relevant energy range for this measurement is from 325 to $1325 \mathrm{MeV}$, which includes $68 \%$ of neutrinos from the BNB.
We report the final cross section result as a function of measured kinematic variables following a "forward-folding" approach. This is done using a migration matrix $S$, which transforms the number of generated events $N_{j}^{\text {gen }}$ in a bin $j$ of generated momentum and angle from any inclusive model to the number of events $N_{i}$ in a bin $i$ of measured momentum and angle. $N_{i}=\sum_{j=1}^{M} S_{i j} N_{j}^{\text {gen }}$, where $S$ is given by $S_{i j}=P($ measured in bin $i \mid$ generated in bin $j)$ and $M$ is the total number of bins.

The efficiency correction as a function of the measured quantities $\tilde{\epsilon}_{i}$ that we applied to our data as described in Eq. (1) is given by

$$
\tilde{\epsilon}_{i}=\frac{\sum_{j=1}^{M} S_{i j} N_{j}^{\mathrm{sel}}}{\sum_{j=1}^{M} S_{i j} N_{j}^{\mathrm{gen}}},
$$

where $N_{j}^{\text {sel }}$ is the number of selected signal events in bin $j$.

The uncertainty on the measurement is dominated by systematic uncertainties, which come from the neutrino flux, neutrino interaction model, and detector response. Uncertainties, both statistical and systematic, are encoded in a covariance matrix $E$. The total uncertainty matrix is a combination of the statistical and systematic errors, $E=E^{\text {stat }}+E^{\text {syst }}$, where $E^{\text {stat }}$ is the statistical uncertainty matrix and $E^{\text {syst }}$ is the systematic covariance matrix.

To assess the uncertainties on the neutrino flux prediction, the final flux simulation from the MiniBooNE Collaboration is utilized [12], updated to the MicroBooNE detector location. For neutrino cross section modeling uncertainties, we use the GENIE framework of event reweighting $[13,20]$ with its standard reweighting parameters. For both cross section and flux systematics we use a multisim technique [21], which consists of generating many Monte Carlo (MC) replicas, each one called a "universe," where parameters in the models are 
varied within their uncertainties. Each universe represents a different reweighting. The simultaneous reweighting of all model parameters allows the correct treatment of correlations among them. $N$ such universes are then created that can be combined to construct the covariance matrix:

$$
E_{i j}=\frac{1}{N} \sum_{n=1}^{N}\left(\sigma_{i}^{n}-\sigma_{i}^{\mathrm{cv}}\right)\left(\sigma_{j}^{n}-\sigma_{j}^{\mathrm{cv}}\right),
$$

where $\sigma$ is a shorthand notation for the double-differential cross section in Eq. (1), $i$ and $j$ correspond to bins in measured quantities, $\sigma_{i}^{\mathrm{cv}}$ is the central value cross section in bin $i$, and $\sigma_{i}^{n}$ is the cross section evaluated in the systematic universe $n$.

A different model is followed for systematics associated with the detector model, as these systematics are dominated by single detector parameters and are not possible to estimate through reweighting. In this case unisim samples [21] are generated, where only one detector parameter at a time is changed by its uncertainty. For $M$ detector parameters, the covariance matrix is

$$
E_{i j}=\sum_{m=1}^{M}\left(\sigma_{i}^{m}-\sigma_{i}^{\mathrm{cv}}\right)\left(\sigma_{j}^{m}-\sigma_{j}^{\mathrm{cv}}\right)
$$

The total flux, cross section, and detector uncertainties amount to $12 \%, 4 \%$, and $16 \%$ of the total cross section, respectively. The largest individual contribution to the detector uncertainty comes from using a simple model to simulate the induced charge on neighboring wires of the TPC, leading to a $13 \%$ uncertainty on the total cross section. Additional uncertainties are assessed on the dirt and simulated $\mathrm{CR}$ background interactions overlaying neutrino interactions, which yield $11 \%$ and $4 \%$ uncertainties on the final cross section measurement, respectively. A summary of systematic uncertainty is shown in Table I.

The double-differential cross section is presented in Fig. 2 and compared with several predictions from different generators. The first uses the default GENIE configuration in GENIE v2.12.10, with the addition of a meson exchange current (MEC) interaction channel modeled with an

TABLE I. Contributions to the total cross section systematic uncertainty.

\begin{tabular}{lc}
\hline \hline Source of uncertainty & Relative uncertainty [\%] \\
\hline Beam flux & 12.4 \\
Cross section modeling & 3.9 \\
Detector response & 16.2 \\
Dirt background & 10.9 \\
Cosmic ray background & 4.2 \\
MC statistics & 0.2 \\
Statistics & 1.4 \\
Total & 23.8 \\
\hline \hline
\end{tabular}

empirical approach [22]. We also compare to the most recent version of GENIE- $\mathrm{v} 3.0 .6$ - in which we use the G18_10a_02_11a comprehensive model configuration. This includes a number of theoretically motivated improvements. It replaces the Bodek-Ritchie Fermi gas nuclear model with a local Fermi gas (LFG) for the nuclear initial state. The Valencia model is used for quasielastic and MEC interactions [23,24], and the Kuzmin-Lyubushkin-Naumov [25] and Berger-Seghal [26] model with form factors from MiniBooNE data [27] for resonant pion production. We also compare to predictions from NUWRO and GIBUU. NUWRO 19.02.1 [28] uses a similar set of models to the GENIE v3.0.6 configuration, though the resonant pion production form factors are modified [29] and the finalstate interaction model is the Oset intranuclear cascade model [30]. GIBUU 2019 [31] has consistent nuclear medium corrections throughout. It also uses a LFG model to describe the nucleon momenta, a separate MEC model [32], and propagates final-state particles according to the Boltzmann-Uehling-Uhlenbeck transport equations.

This is the first test of neutrino event generators against double-differential neutrino scattering data on argon. As is also seen in comparisons to neutrino data on carbon [33,34], high $\chi^{2}$ values between data and predictions are observed taking into account the full covariance matrix with off-diagonal elements (not displayed in Fig. 2). The largest disagreements between the data and predictions are observed in the high-momentum bins in the most forwardgoing muon angular bins of $0.94 \leq \cos \theta_{\mu} \leq 1$ and $0.86 \leq \cos \theta_{\mu}<0.94$. This region strongly disfavors the GENIE v2 with empirical MEC. Other predictions show less tension with the data in this phase space with the exception of the highest momentum bin with the angular range of $0.86 \leq \cos \theta_{\mu}<0.94$. The lowest $\chi^{2}$ value is obtained for the GENIE v3 model with a $\chi^{2}$ of 103.9 for 42 bins. The reduced tension originates from the overall reduced cross section in the forward direction when adopting the local Fermi gas nuclear initial state, which is expected to be a more realistic momentum distribution of the initial state nucleons, and to a lesser extent the random phase approximation (RPA) correction as included in the GENIE v3 and NUWRO predictions. These effects have the largest impact at low neutrino energies and for heavy nuclear targets, which explains why MicroBooNE is more sensitive to these effects than previous experiments. A perfect backscattered $\mathrm{CC}$ quasielastic muon at 800 (1325) $\mathrm{MeV}$ neutrino energy corresponds to the square of the four-momentum transfer $Q^{2}$ of 2.5(7.0) $\mathrm{GeV}^{2}$. For this reason, these new MicroBooNE cross section results are very valuable for addressing and testing the details of the current neutrino cross section models and for making progress in understanding the physics associated with neutrino interactions. Smearing and covariance matrices, as well as cross section and flux tabulated values, are available in the Supplemental Material [35]. 

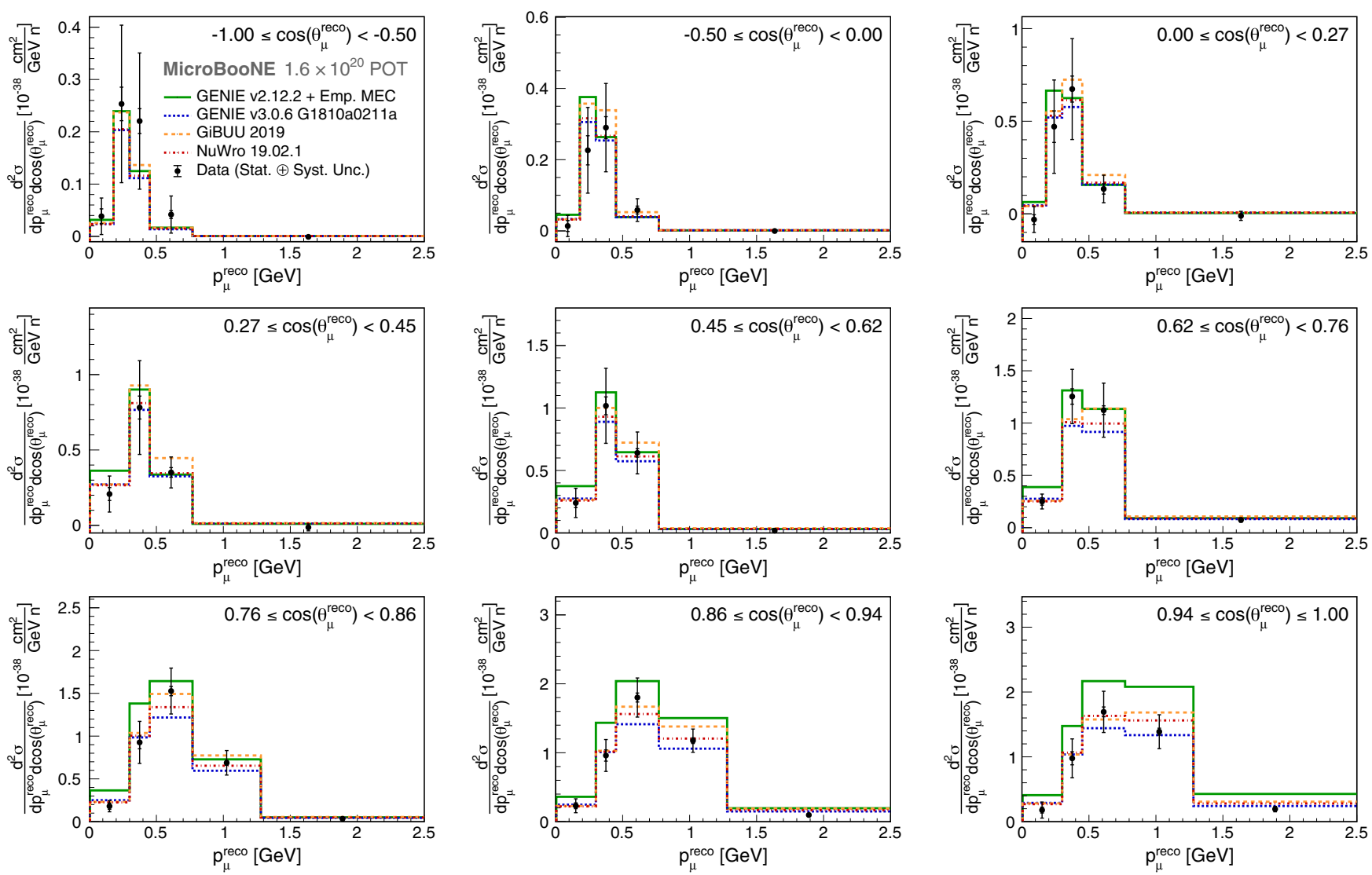

FIG. 2. $\nu_{\mu}$ CC inclusive double-differential cross section on argon per nucleon $n$ as a function of the measured muon momentum and cosine of the measured muon polar angle (angle with respect to the incoming neutrino direction), $d^{2} \sigma /\left(d p_{\mu}^{\text {reco }} d \cos _{\mu}^{\text {reco }}\right)\left[10^{-38} \mathrm{~cm}^{2} /\right.$ $(\mathrm{GeV} n)]$. The data (black) are compared to a GENIE v2 with empirical MEC prediction (green), a GENIE v3 prediction (blue), a GIBUU prediction (orange), and a NUWRO prediction (red), as described in the text. The vertical bars show statistical and systematic uncertainties.

Additionally, we compute a flux-integrated cross section $\sigma\left(\nu_{\mu}+\mathrm{Ar} \rightarrow \mu^{-}+X\right)$ per nucleon of

$$
\sigma=0.693 \pm 0.010 \text { (stat) } \pm 0.165 \text { (syst) } \times 10^{-38} \mathrm{~cm}^{2}
$$

which is obtained by integrating the number of signal and background events, as well as the efficiency over all bins. The measured flux-integrated cross section agrees with the predictions from the models described above within uncertainty, with GENIE v2 giving the largest discrepancy.

In summary, we have reported the first double-differential $\nu_{\mu}$ charged current inclusive cross section on argon. The presented analysis has full angular coverage and uses multiple Coulomb scattering to estimate the muon momentum, a significant step forward for the LArTPC technology.

As shown in the comparison with various predictions, these data provide a way to differentiate models in neutrino event generators. These measurements not only inform the theory of neutrino-nucleus scattering, but also reduce the systematic uncertainties associated with cross section measurements in neutrino oscillation experiments.
This document was prepared by MicroBooNE using the resources of the Fermi National Accelerator Laboratory (Fermilab), a U.S. Department of Energy, Office of Science, HEP User Facility. Fermilab is managed by Fermi Research Alliance, LLC (FRA), acting under Contract No. DE-AC02-07CH11359. MicroBooNE is supported by the U.S. Department of Energy, Office of Science, Offices of High Energy Physics and Nuclear Physics; the U.S. National Science Foundation; the Swiss National Science Foundation; the Science and Technology Facilities Council (STFC), part of UK Research and Innovation; and The Royal Society (United Kingdom). Additional support for the laser calibration system and CR tagger was provided by the Albert Einstein Center for Fundamental Physics, Bern, Switzerland.

*microboone_info@fnal.gov

[1] B. Abi et al. (DUNE Collaboration), The DUNE far detector interim design report, Volume 1: Physics, technology and strategies, arXiv:1807.10334. 
[2] B. Abi et al. (DUNE Collaboration), The DUNE far detector interim design report, Volume 2: Single-phase module, arXiv:1807.10327.

[3] B. Abi et al. (DUNE Collaboration), The DUNE far detector interim design report, Volume 3: Dual-phase module, arXiv:1807.10340.

[4] M. Antonello et al. (LAr1-ND, ICARUS-WA104, and MicroBooNE Collaborations), A proposal for a three detector short-baseline neutrino oscillation program in the Fermilab Booster neutrino beam, arXiv:1503.01520.

[5] R. Acciarri et al. (ArgoNeuT Collaboration), Measurements of inclusive muon neutrino and antineutrino charged current differential cross sections on argon in the NuMI antineutrino beam, Phys. Rev. D 89, 112003 (2014).

[6] C. Anderson et al. (ArgoNeuT Collaboration), First Measurements of Inclusive Muon Neutrino Charged Current Differential Cross Sections on Argon, Phys. Rev. Lett. 108, 161802 (2012).

[7] J. A. Formaggio and G. P. Zeller, From eV to EeV: Neutrino cross sections across energy scales, Rev. Mod. Phys. 84, 1307 (2012).

[8] P. Abratenko et al., Determination of muon momentum in the MicroBooNE LArTPC using an improved model of multiple Coulomb scattering, J. Instrum. 12, P10010 (2017).

[9] R. Acciarri et al. (MicroBooNE Collaboration), Design and construction of the MicroBooNE detector, J. Instrum. 12, P02017 (2017).

[10] R. Acciarri et al. (MicroBooNE Collaboration), Noise characterization and filtering in the MicroBooNE liquid argon TPC, J. Instrum. 12, P08003 (2017).

[11] J. Conrad, B. Jones, Z. Moss, T. Strauss, and M. Toups, The photomultiplier tube calibration system of the MicroBooNE experiment, J. Instrum. 10, T06001 (2015).

[12] A. A. Aguilar-Arevalo et al. (MiniBooNE Collaboration), Neutrino flux prediction at MiniBooNE, Phys. Rev. D 79, 072002 (2009).

[13] C. Andreopoulos et al., The GENIE neutrino Monte Carlo generator, Nucl. Instrum. Methods Phys. Res., Sect. A 614, 87 (2010), version 2_12_2.

[14] D. Heck, J. Knapp, J. N. Capdevielle, G. Schatz, and T. Thouw, CORSIKA: A Monte Carlo code to simulate extensive air showers, Forschungszentrum Karlsruhe Report FZKA, Report No. FZKA-6019, 1998, https://www.ikp.kit .edu/corsika, version 7.4003 with constant mass composition model.

[15] S. Agostinelli et al., Geant4-a simulation toolkit, Nucl. Instrum. Methods 506, 250 (2003).

[16] R. Pordes and E. Snider, The liquid argon software toolkit (LArSoft): Goals, status and plan, Proc. Sci., ICHEP2016 (2016) 182.

[17] B. Baller, Liquid argon TPC signal formation, signal processing and reconstruction techniques, J. Instrum. 12, P07010 (2017).

[18] R. Acciarri et al. (MicroBooNE Collaboration), The Pandora multi-algorithm approach to automated pattern recognition of cosmic-ray muon and neutrino events in the MicroBooNE detector, Eur. Phys. J. C 78, 82 (2018).

[19] C. Adams et al. (MicroBooNE Collaboration), Calibration of the charge and energy response of the MicroBooNE liquid argon time projection chamber using muons and protons, arXiv:1907.11736.

[20] C. Andreopoulos et al., The GENIE neutrino Monte Carlo generator: Physics and user manual, 2015, arXiv: 1510.05494.

[21] B. P. Roe, Statistical errors in Monte Carlo estimates of systematic errors, Nucl. Instrum. Methods 570, 159 (2007).

[22] T. Katori, Meson exchange current (MEC) models in neutrino interaction generators, AIP Conf. Proc. 1663, 030001 (2015).

[23] J. Nieves, I. R. Simo, and M. J. Vicente Vacas, Inclusive charged-current neutrino-nucleus reactions, Phys. Rev. C 83, 045501 (2011).

[24] R. Gran, J. Nieves, F. Sanchez, and M. J. Vicente Vacas, Neutrino-nucleus quasi-elastic and $2 \mathrm{p} 2 \mathrm{~h}$ interactions up to 10 GeV, Phys. Rev. D 88, 113007 (2013).

[25] K. S. Kuzmin, V. V. Lyubushkin, and V. A. Naumov, Lepton polarization in neutrino-nucleon interactions, Mod. Phys. Lett. A 19, 2815 (2004).

[26] C. Berger and L. M. Sehgal, Partially conserved axial vector current and coherent pion production by low energy neutrinos, Phys. Rev. D 79, 053003 (2009).

[27] J. A. Nowak, Four momentum transfer discrepancy in the charged current $\pi^{\text {plus }}$ production in the MiniBooNE: Data vs. theory, AIP Conf. Proc. 1189, 243 (2009).

[28] T. Golan, J. Sobczyk, and J. Zmuda, NuWro: the Wrocław Monte Carlo generator of neutrino interactions, Nucl. Phys. B229-B232, 499 (2012).

[29] K. M. Graczyk, D. Kiełczewska, P. Przewłocki, and J. T. Sobczyk, $C_{5}^{A}$ axial form factor from bubble chamber experiments, Phys. Rev. D 80, 093001 (2009).

[30] L. Salcedo, E. Oset, M. Vicente-Vacas, and C. Garcia-Recio, Computer simulation of inclusive pion nuclear reactions, Nucl. Phys. A484, 557 (1988).

[31] O. Buss, T. Gaitanos, K. Gallmeister, H. van Hees, M. Kaskulov, O. Lalakulich, A. B. Larionov, T. Leitner, J. Weil, and U. Mosel, Transport-theoretical description of nuclear reactions, Phys. Rep. 512, 1 (2012).

[32] K. Gallmeister, U. Mosel, and J. Weil, Neutrino-induced reactions on nuclei, Phys. Rev. C 94, 035502 (2016).

[33] D. Ruterbories et al. (MINERvA Collaboration), Measurement of quasielastic-like neutrino scattering at $\left\langle E_{\nu}\right\rangle \sim$ $3.5 \mathrm{GeV}$ on a hydrocarbon target, Phys. Rev. D 99, 012004 (2019).

[34] K. Abe et al. (T2K Collaboration), Measurement of inclusive double-differential $\nu_{\mu}$ charged-current cross section with improved acceptance in the T2K off-axis near detector, Phys. Rev. D 98, 012004 (2018).

[35] See Supplemental Material at http://link.aps.org/ supplemental/10.1103/PhysRevLett.123.131801 for tabulated values of cross section and flux, as well as smearing and covariance matrices. 